ized in cells capable of enlargement even before they are stimulated to greater enlargement by IAA. However, hydroxyproline had no effect on the subsequent ability of the cells to enlarge.

There may be a further mechanism of hydroxyproline inhibition. Using sycamore callus tissue, Holleman (Proc. US Nat. Acad. Sci., 5\%, 50; 1967) showed that the hydroxyproline in the wall protein arose from hydroxylation of previously incorporated proline (as in collagen). In the presence of an inhibitor of hydroxylation ( $\alpha \alpha^{\prime}$-dipyridyl) hydroxyproline added to the cells was still incorporated into the cell wall protein. Holleman suggests that this incorporated hydroxyproline has forced its way into the protein which is then rendered non-functional, and in this way causes the cell growth inhibition. But what is the function of this protein? Although it is implicated with cell enlargement, Cleland and Karlsnes (Plant Physiol., 42, 669; 1967) also implicate it with the cessation of enlargement. Their conclusion is based on the finding that in cell walls of growing pea epicotyls, the greatest increase in the protein which contains hydroxyproline occurs when cell elongation has finished. The two systems may not be incompatible but certainly there are no clear-cut answers yet. More data are needed on the types of protein required for cell growth and on the mechanism by which IAA influences these, and also how this hydroxyproline-containing protein fits into the cell wall during enlargement.

This protein, extensin, has often been likened to collagen and indeed collagen has now been reported by De and Ghosh (Exp. Cell Res., 47, 637; 1967) to be present in plant cells. But surprisingly it is in the nucleus.

\section{Sulphur in RNA}

\section{from our Molecular Biology Correspondent}

THe discovery of sulphydryl groups in transfer RNA has increased the number and variety of "unusual" bases recognized in these species. Their function, though the subject of some slightly remote speculation, is unknown. The very unexpectedness of the thiolated bases and their chemical reactivity, which offers the possibility of generating covalent cross-links, makes them, however, a source of considerable interest.

There appear to be several such bases, but one of them, 4-thiouracil, is the most prevalent. In total $t$ RNA from $E$. coli there appear to be rather fewer than two sulphur atoms per molecule. Lipsett has now shown (J. Biol. Chem., 242, 4067; 1967) that, as expected, mild oxidation converts the thiol groups into disulphide bonds, and from a complete nuclease digest of $t$ RNA a 4-thioUMP disulphide has been isolated. No mixed disulphides with any other thiolated nucleotides are found. In a further study Lipsett and Doctor (ibid., 4072) have examined a single $t$ RNA, with a greater than average thiol content. This is $E$. coli tyrosine $t$ RNA-one of the easier species to prepare, since it appears at one end of the countercurrent distribution profile. Titrations with thiol reagents show that two sulphydryl groups are present, and both turn out to be 4-thioUMP. On reduction, a disulphide bridge is formed as before, and it is surprising to find that the sedimentation coefficient is not increased. It follows that the disulphide bridge is intramolecular. This is a useful (as well as an unexpected) result, for it indicates that none of the dimeric, and other aggregated, species which have been observed by other workers are likely to involve intermolecular disulphide bonds.

This internal disulphide formation is accompanied by a small but significant increase in absorbance and a decrease in thermal stability. It therefore seems likely that the new bond is achieved at the expense of some base-pairing. At the same time the amino-acid acceptor activity survives intact, despite the evident disturbance of the conformation. (It has not so far been established whether other functions, such as the capacity to attach to the ribosome, are extirpated).

The 4-thioUMP has an absorption band at about $330 \mathrm{~m} \mu$, which is therefore resolved from the main nucleotide absorption centred at $260 \mathrm{~m} \mu$. Although its concentration is low, and the absorption therefore weak, there is clear indication of hypochromicity in this band, which supports the view that the 4. thioUMP forms part of the double-helical structure. After oxidation the effect is lost, although a shifted long-wavelength absorption can still be seen.

That the 4-thiouracil can indeed pair with adenine in a helical structure has just been demonstrated by Scheit (Biochim. Biophys. Acta, 145, 535, 1967), who has prepared copolymers of UMP and 4-thioUMP, and complexed them with poly A. Hypochromism is observed both at 260 and $330 \mathrm{~m} \mu$-where the thionucleotide absorbs. Only three-stranded complexes appear to be formed at room temperature, but temperature-absorbance profiles show two phases, which by analogy with the intensively studied poly $(\mathrm{A}+2 \mathrm{U})$ system suggests that in a certain temperature range the two-stranded form is stable. It is clear, however, that the A-thioU pairing is much weaker than A-U; this is consistent with the observations of Lipsett and Doctor on the apparent readiness with which basepairs involving 4-thioUMP are broken to allow the formation of internal disulphide bonds.

One would hesitate at this juncture to hazard any guesses at the possible purpose of thionucleotides in in $t$ RNA. It is nevertheless perhaps interesting to store the information alongside the recently demonstrated effects of sulphydryl-blocking reagents on the function of $E$. coli ribosomes (see, for example, Traut and Haenni, Europ. J. Biochem., 2, 64; 1967).

\section{They Wobble}

\section{from our Cell Biology Correspondent}

In the latest two papers from Khorana's laboratory, Söll, Cherayil and Bock, and Söll and RajBhandry (J. Mol. Biol., 29, 97 and 113; 1967), summarize their results on the coding specificities of yeast and $E$. coli $t$ RNA. Their data are in striking agreement with Crick's (1966) Wobble hypothesis.

Because the genetic code is highly degenerate, Crick predicted that one species of $t$ RNA can recognize more than one codon. An unambiguous demonstration of this requires homogeneous $t$ RNA and a technique to study interactions. But now between codons and anti-codons $t$ RNA species can be isolated in a sufficiently pure form to allow meaningful experiments, and Nirenberg and Leder (1964) have found that the interaction can be detected by binding $t \mathrm{RNA}$ to ribosomes programmed with tri-ribonucleotides (single codons). The synthesis, in Khorana's laboratory, of 